\title{
EGaln Fiber Enabled Highly Flexible Supercapacitors
}

\author{
Minghui Duan, Yi Ren, Xuyang Sun, Xiyu Zhu, Xuelin Wang, Lei Sheng, and Jing Liu*
}

Cite This: ACS Omega 2021, 6, 24444-24449

Read Online
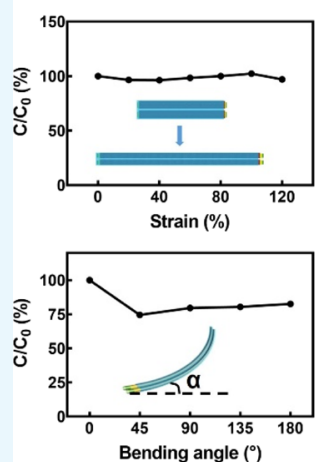
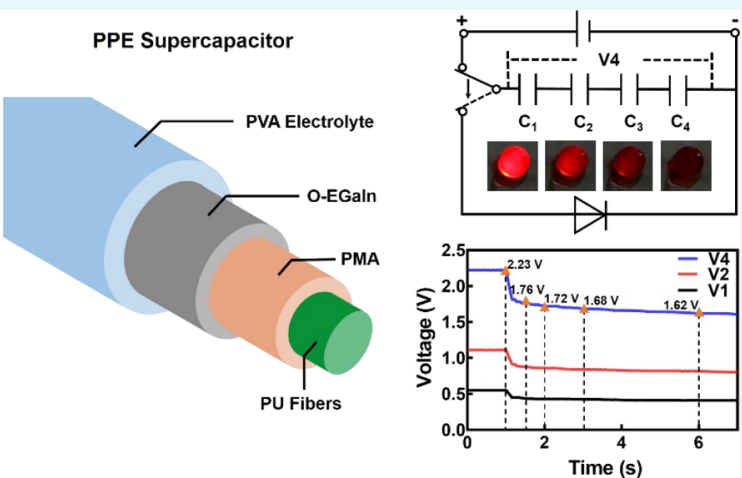

ABSTRACT: Attributed to their soft and stretchable feature, flexible supercapacitors have attracted increasing attention in areas of soft electronics, wearable devices, and energy storage systems. However, it is a challenge to manufacture all-soft supercapacitors with highly flexible properties and excellent electrochemical performance. Here, an EGaIn-based fibrous supercapacitor, which is composed of two paralleled stretchable fibers, is designed and demonstrated first with flexible and stretchable properties. EGaIn coated on the surface of polyurethane (PU)@polymethacrylate (PMA) fibers can serve as a current collector. The prepared supercapacitor is measured with an areal specific capacitance of $26.71 \mathrm{mF} \cdot \mathrm{cm}^{-2}$ by mixing $\mathrm{Fe}_{3} \mathrm{O}_{4}$ microparticles with EGaIn. This value can increase up to $61.34 \mathrm{mF} \cdot \mathrm{cm}^{-2}$ after vacuum pumping, the mechanism of which is further revealed to be related with the coarser surface and airhole formation on the fibers. The supercapacitor maintains an excellent electrochemical performance when stretched to $120 \%$ strain and exhibits a long cycling life through a charge-discharge cycle of over 1000 times. Finally, the supercapacitors are adopted to light the LED, demonstrating that those supercapacitors can work successfully. All these characteristics indicate the huge potential of EGaIn-based supercapacitors in the field of flexible electronics and wearable devices.

\section{INTRODUCTION}

The development of soft electronics and wearable devices are attracting more and more interest due to their potential applications on health monitoring and motion tracking. ${ }^{1-5}$ Stretchable and flexible sensors, circuits, and soft energy storage devices are indispensable to the performance of wearable devices. $^{6-9}$ The production of high-performance soft energy storage devices are considered as the challenging part in wearables. ${ }^{10}$ Flexible supercapacitors that have excellent energy storage performance can be used as soft energy storage devices. In fact, supercapacitors, which were first introduced in 1957 , have been widely applied as energy storage devices. ${ }^{11}$ They are distinguished for their fast charging and discharging processes, great capacitance, and ultralong life span. ${ }^{12,13}$ Higher power density and energy density are also obtained simultaneously. ${ }^{14,15}$ These excellent electrical properties make supercapacitors stand out from diverse energy storage devices. What is more, supercapacitors are considered to be a potential alternative to conventional lithium ion batteries to solve the limitations of large sizes and heavy weights. ${ }^{16}$
In order to improve the performance of flexible supercapacitors, various methods have been explored, including optimizations on the designs and materials. Generally, flexible supercapacitors include fibrous supercapacitors, planar supercapacitors, etc. ${ }^{17-21}$ The electrodes of fibrous supercapacitors can be placed in the form of parallel, spiral or coaxial structures. Compared to planar supercapacitors, fibrous supercapacitors show better flexibility and usually exhibit high stretchability for wearables. ${ }^{18}$ Besides, the electrode materials are influential as well. Carbon-based materials have been widely used, like carbon nanofibers and graphene. ${ }^{17-22}$ Advantages of carbon nanomaterials are that they have high specific surface area and good flexibility. However, the conductivity of carbon-based materials is inferior to metallic

Received: $\quad$ May 31, 2021

Published: September 17, 2021 
materials. And the fabrication of supercapacitors should be further simplified and standardized. Finally, Metal oxide microparticles, such as $\mathrm{MnO}_{2}$ and $\mathrm{Fe}_{3} \mathrm{O}_{4}$, which can increase effective facing area of supercapacitors and are also verified pseudo-capacitive materials, have been commonly used to improve the specific capacitance of supercapacitors. ${ }^{17,18}$

Recently, liquid metal, a new emerging material for soft electronics, is attracting increasing interest because it can provide not only high conductivity but also perfect flexibility. ${ }^{23,24}$ Studies on flexible electronics based on liquid metals include flexible conductors, antennas, transistors, and sensors, as well as soft supercapacitors and batteries. ${ }^{25-33}$ For instance, Park et al. fabricated a stretchable supercapacitor and integrated it with a strain sensor using liquid metal. ${ }^{30}$ This system was proven to work well for real-time health monitoring. Furthermore, Kim et al. designed a planar supercapacitor with liquid metal electrodes. ${ }^{31}$ Carbon nanotubes were uniformly deposited on the surface of liquid metal electrodes to improve the capacitance. The highest areal capacitance of supercapacitors fabricated by this method was about $12.4 \mathrm{mF} \cdot \mathrm{cm}^{-2}$. At this stage, the supercapacitors could only remain stable under $30 \%$ strain. Overall, despite the advances, difficulty remains in designing supercapacitors with high capacitance, stability, and flexibility.

In this work, we attempted to design all-soft fiber supercapacitors with high flexibility and capacitance based on eutectic gallium-indium alloy (EGaIn) and metal oxide microparticles. Fibers applied in this experiment were made of polyurethane (PU). EGaIn was coated onto the surface of polyurethane fibers with the help of polymethacrylate (PMA). The obtained PU@PMA@EGaIn (PPE) fibers were then placed parallel to each other to form supercapacitors. To obtain higher capacitance, metal oxide was introduced, including $\mathrm{MnO}_{2}$ and $\mathrm{Fe}_{3} \mathrm{O}_{4}$ microparticles. In this article, EGaIn mixed with metal oxide microparticles was named as $\mathrm{O}$ EGaIn. The results showed that both $\mathrm{MnO}_{2}$ and $\mathrm{Fe}_{3} \mathrm{O}_{4}$ microparticles can greatly improve the capacitance, especially for $\mathrm{Fe}_{3} \mathrm{O}_{4}$ microparticles. The highest specific capacitive value we obtained is about $61.34 \mathrm{mF} \cdot \mathrm{cm}^{-2}$. The supercapacitors could tolerate tensile strain of up to $120 \%$. It is expected that liquid metal will show more advantages in the development of next-generation flexible supercapacitors.

\section{RESULTS AND DISCUSSION}

Figure 1 illustrates the fabrication and the characterization of flexible PPE supercapacitors (PPE SCs). As shown in Figure 1a, the PPE SCs were composed of four layers, including PU fibers, PMA, O-EGaIn, and PVA electrolyte. The preparation of O-EGaIn is described in Figure S1. The fabrication process of PPE SCs is illustrated in Figure 1b. First, PU fibers were coated by PMA and O-EGaIn successively. The utilization of PMA can enhance the adhesion force between O-EGaIn and fibers, which was tested later. Afterward, two PPE fibers were placed parallelly and coated by $\mathrm{NaCl}-\mathrm{CaCl}_{2}$ PVA gel electrolyte. Generally, the whole fabrication process is rather simple. Figure 1c shows the pictures of fibers and the supercapacitor during the fabrication process.

The surface morphologies and structures of the PPE fibers, PPE- $\mathrm{MnO}_{2}$ fibers, and PPE- $\mathrm{Fe}_{3} \mathrm{O}_{4}$ fibers were observed and compared by scanning electron microscopy (SEM) and energy dispersive spectrometry (EDS). Coated by EGaIn, the surface of the fiber is smooth and flat as shown in Figures S2 and S3a. Meanwhile, $\mathrm{PPE}-\mathrm{MnO}_{2}$ fibers and $\mathrm{PPE}-\mathrm{Fe}_{3} \mathrm{O}_{4}$ fibers have (a)

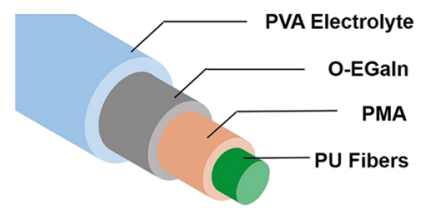

(c)

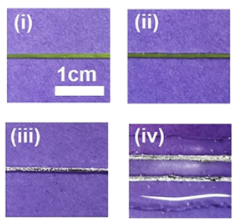

(b)
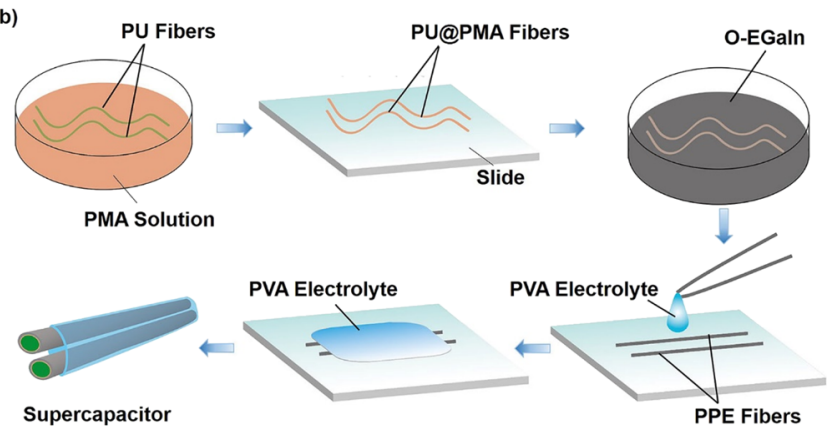

(d)
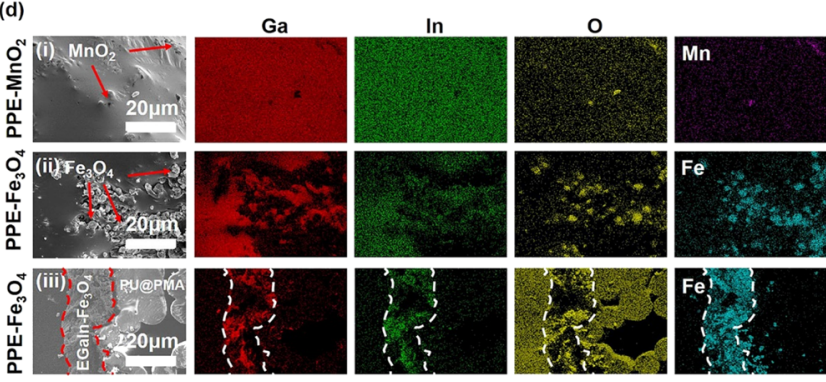

Figure 1. Fabrication and the characterization of PPE SCs: (a) the schematic structure of PPE SCs; (b) the fabrication process of PPE SCs; (c) photos of the fibers and the supercapacitor: (i) PU fiber, (ii) PU@PMA fiber, (iii) PPE fiber, and (iv) the supercapacitor; and (d) SEM and EDS mapping images of the structure of $\mathrm{PPE}-\mathrm{MnO}_{2}$ and

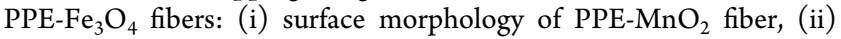
surface morphology of $\mathrm{PPE}-\mathrm{Fe}_{3} \mathrm{O}_{4}$ fiber, and (iii) the partial structure of the cross profile of $\mathrm{PPE}-\mathrm{Fe}_{3} \mathrm{O}_{4}$ fiber.

coarser surfaces as shown in Figure $\mathrm{S} 3 \mathrm{~b}-\mathrm{d}$. In Figure 1d-i,ii presents the surface structures of PPE- $\mathrm{MnO}_{2}$ and $\mathrm{PPE}-\mathrm{Fe}_{3} \mathrm{O}_{4}$ fibers, respectively, and panel d-iii shows the cross profile of PPE- $\mathrm{Fe}_{3} \mathrm{O}_{4}$ fiber. The $\mathrm{MnO}_{2}$ and $\mathrm{Fe}_{3} \mathrm{O}_{4}$ microparticles were deposited on the surface of fibers in a large area, and the coarser surface suggested a larger effective facing area of supercapacitors.

Through repeated experiments, we found that EGaIn would react with acidic or alkaline PVA gel electrolyte. Thus, the neutral electrolyte was chosen to prepare the supercapacitor. Considering that the solubility of $\mathrm{CaCl}_{2}$ is relatively higher than $\mathrm{NaCl}$ and the existence of $\mathrm{CaCl}_{2}$ can avoid excessive water loss, we added both $\mathrm{NaCl}$ and $\mathrm{CaCl}_{2}$ together to improve the ion concentration and mechanical performance of PVA gel electrolyte. $\mathrm{NaCl}-\mathrm{CaCl}_{2}$ PVA electrolyte with different doping ratios were prepared and tested. Results are presented in Figure 2a. Obviously, with the increasing proportion of $\mathrm{NaCl}$, the solution became opaque gradually. When the percentage of $\mathrm{NaCl}$ increased up to $100 \%$, it failed to form uniform solution with the precipitation of some flocculent precipitates. On the other hand, when the percentage of $\mathrm{CaCl}_{2}$ is $100 \%$, the solution is too sticky to form a gel electrolyte film. Thus, to obtain good mechanical properties and higher ionic conductivity, the most suitable proportion of $\mathrm{NaCl}$ is around 

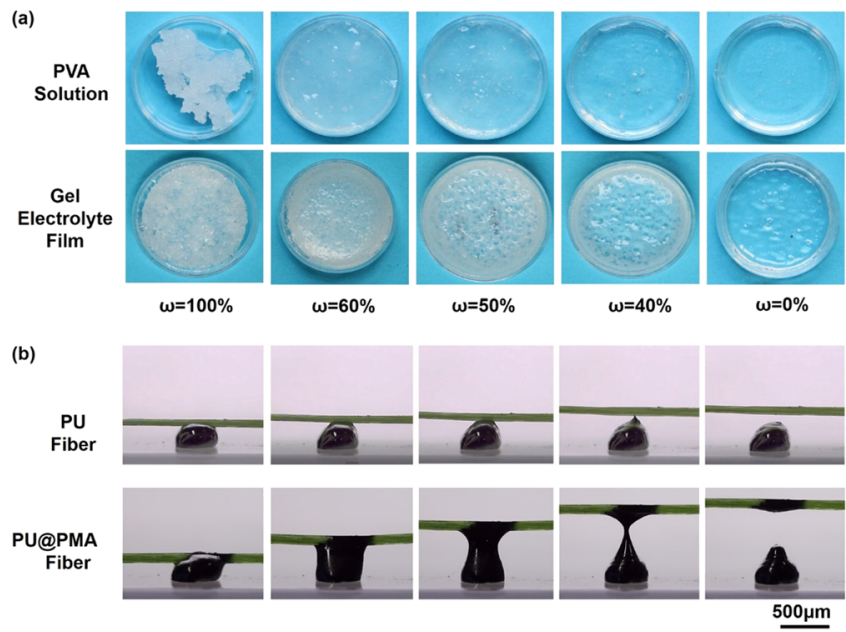

(c)

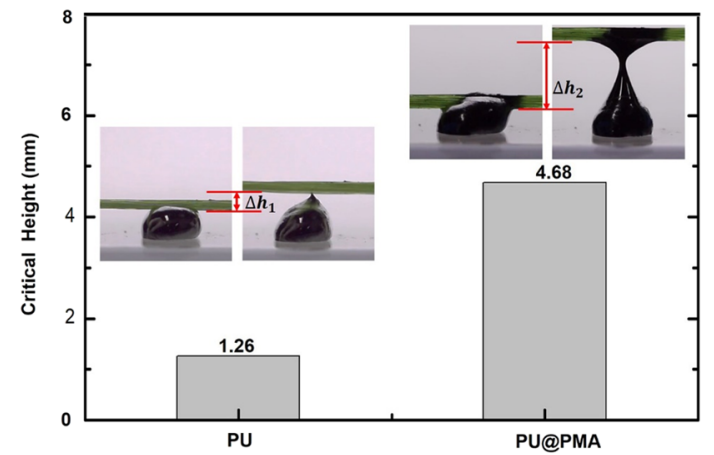

Figure 2. Characterization of PVA gel and PPE fibers. (a) Comparison of $\mathrm{NaCl}-\mathrm{CaCl}_{2}$ PVA gel with different mixing ratios, and $\omega$ is the mass fraction of $\mathrm{NaCl}$; (b) comparison of the EGaIn's adhesion to PU fiber and PU@PMA fiber; (c) the critical height when the EGaIn droplet was separated from PU and PU@PMA fibers.

40-60\%. In our experiment, the mixing ratio between $\mathrm{NaCl}$ and $\mathrm{CaCl}_{2}$ in PVA gel adopted was 2:3.

Guo et al. found that PMA caused high adhesion with EGaIn because of the hydrogen bonding formed between PMA and EGaIn liquid metal oxide. ${ }^{34}$ The function of PMA was tested by an adhesion experiment, as shown in Figure 2b. PU@PMA fibers were obtained by immersing PU fibers in PMA solution. The PU fiber and PU@PMA fiber were pressed onto the EGaIn droplet and then lifted up tardily. Obviously, EGaIn was more adhesive to the PU@PMA fiber than PU fiber. EGaIn could reach a higher position with the PU@PMA fiber. Moreover, when the fibers and EGaIn were separated, some EGaIn stuck to the PU@PMA fiber. However, almost no EGaIn remained on the PU fiber. Figure $2 c$ depicts the critical height before EGaIn was separated from the fibers. The height of PU@PMA fiber was 4.68 mm, which was 3.7 times higher than that of PU fiber. Thus, it is concluded that PMA can greatly enhance the adhesion between EGaIn and fibers.

To test the electrochemical performance of the supercapacitor made of PPE, we first investigated the cyclic voltammetry $(\mathrm{CV})$ response of the supercapacitor in a twoelectrode electrochemical cell. The CV curves of PPE SC, PPE$\mathrm{MnO}_{2}$ SC, $\mathrm{PPE}-\mathrm{Fe}_{3} \mathrm{O}_{4}$ SC, and $\mathrm{PPE}-\mathrm{Fe}_{3} \mathrm{O}_{4} \mathrm{SC}$ (after vacuum freeze-drying) were all measured for comparison as shown in Figure 3a. At a scan rate of $5 \mathrm{mV} \cdot \mathrm{s}^{-1}$, the areal specific capacitance of the $\mathrm{PPE}-\mathrm{Fe}_{3} \mathrm{O}_{4} \mathrm{SC}$ was $26.71 \mathrm{mF} \cdot \mathrm{cm}^{-2}$. However, the $\mathrm{PPE}-\mathrm{Fe}_{3} \mathrm{O}_{4} \mathrm{SC}$ (vacuum) showed an areal specific capacitance of $61.34 \mathrm{mF} \cdot \mathrm{cm}^{-2}$, which was 2.30 times
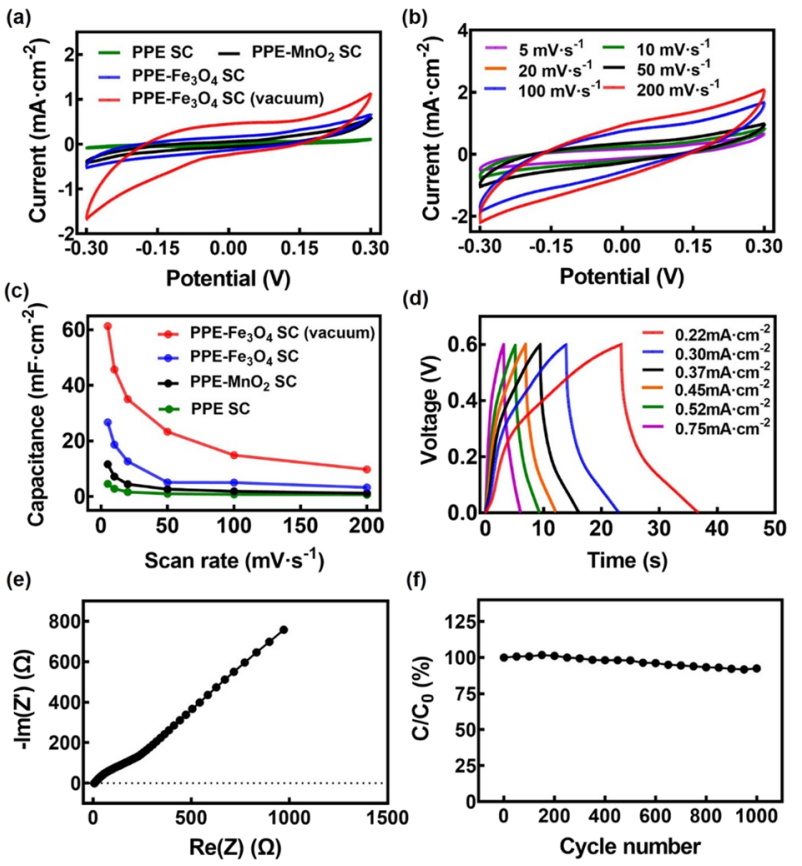

Figure 3. Electrochemical test. (a) Cyclic voltammetry (CV) curves of PPE SC, PPE- $\mathrm{MnO}_{2}$ SC, PPE- $\mathrm{Fe}_{3} \mathrm{O}_{4} \mathrm{SC}$, and PPE- $\mathrm{Fe}_{3} \mathrm{O}_{4} \mathrm{SC}$ (after vacuum freeze-drying) at a scan rate of $5 \mathrm{mV} \cdot \mathrm{s}^{-1}$; (b) CV curves of the $\mathrm{PPE}-\mathrm{Fe}_{3} \mathrm{O}_{4} \mathrm{SC}$ at different scan rates; (c) plots of areal specific capacitances of the four kinds of SCs above as the scan rate increases; (d) galvanostatic charge-discharge curves measured at different current densities; (e) the Nyquist plot of the PPE- $\mathrm{Fe}_{3} \mathrm{O}_{4} \mathrm{SC}$; (f) the cyclic performance of the $\mathrm{PPE}-\mathrm{Fe}_{3} \mathrm{O}_{4} \mathrm{SC}$. $\mathrm{C}_{0}$ and $\mathrm{C}$ correspond to specific capacitances at the initial and subsequent cycles, respectively.

that of the PPE- $\mathrm{Fe}_{3} \mathrm{O}_{4} \mathrm{SC}, 5.30$ times that of the PPE- $\mathrm{MnO}_{2}$ SC, and 13.57 times that of the PPE SC. The nearly rectangular shape is actually a characteristic of the pseudocapacitance of $\mathrm{MnO}_{2}$ and $\mathrm{Fe}_{3} \mathrm{O}_{4} \cdot{ }^{35,36}$ To evaluate why the areal specific capacitances of $\mathrm{PPE}-\mathrm{Fe}_{3} \mathrm{O}_{4} \mathrm{SCs}$ (vacuum) are higher than $\mathrm{PPE}-\mathrm{Fe}_{3} \mathrm{O}_{4}$ SCs, SEM images were observed, as shown in Figure S4. A coarser surface than $\mathrm{PPE}-\mathrm{Fe}_{3} \mathrm{O}_{4} \mathrm{SC}$ and many airholes were found on the surface of $\mathrm{PPE}-\mathrm{Fe}_{3} \mathrm{O}_{4}$ fiber after $\mathrm{PPE}-\mathrm{Fe}_{3} \mathrm{O}_{4} \mathrm{SCs}$ were vacuum pumped. To calculate the capacitance of fibrous supercapacitors, we can treat them as a parallel plate capacitor approximately. Thus, the capacitance of PPE SCs can be calculated according to the following equation:

$$
C=\frac{\varepsilon S}{d}
$$

where $C$ is the capacitance and $\varepsilon$ is the dielectric constant of the PVA gel. $S$ and $d$ are the effective facing area and distance of the PPE fibers. The coarser surface and airholes of PPE$\mathrm{Fe}_{3} \mathrm{O}_{4} \mathrm{SCs}$ (vacuum) led to larger $S$; therefore, the capacitance was much higher. However, the capacitance of PPE- $\mathrm{Fe}_{3} \mathrm{O}_{4} \mathrm{SCs}$ (vacuum) after being measured repeatedly was not steady, so the PPE- $\mathrm{Fe}_{3} \mathrm{O}_{4} \mathrm{SCs}$ had better performance.

Figure $3 \mathrm{~b}$ shows the $\mathrm{CV}$ curves of the $\mathrm{PPE}-\mathrm{Fe}_{3} \mathrm{O}_{4} \mathrm{SC}$ at different scan rates. We measured six $\mathrm{CV}$ curves at a scan rate from 5 to $200 \mathrm{mV} \cdot \mathrm{s}^{-1}$, which show the gradually increasing area and similar shape. According to pre-experiment results, the range of scan voltage was determined from -0.3 to $0.3 \mathrm{~V}$. We obtained the CV curves of other SCs using the same parameter in Figure S5 $(a, b)$. The areal specific capacitance, 
which was calculated from CV curves, differed regularly when the scan rate changed. As shown in Figure 3c, the areal specific capacitance of the $\mathrm{PPE}-\mathrm{Fe}_{3} \mathrm{O}_{4} \mathrm{SC}$ reduced gradually from 26.71 to $3.24 \mathrm{mF} \cdot \mathrm{cm}^{-2}$ when the scan rate varied from 5 to 200 $\mathrm{mV} \cdot \mathrm{s}^{-1}$, with a reduction rate of $87.9 \%$. In addition, because the upper and lower areas of the $X$ axis were not the same, the integration result was divided to calculate the Coulomb efficiency (CE) during the charge and discharge processes. At a scan rate of $5 \mathrm{mV} \cdot \mathrm{s}^{-1}$, the Coulomb efficiency of the PPE$\mathrm{Fe}_{3} \mathrm{O}_{4} \mathrm{SC}$ was $99.66 \%$, which indicated that leakage current of the $\mathrm{PPE}-\mathrm{Fe}_{3} \mathrm{O}_{4} \mathrm{SC}$ was low.

The galvanostatic charge-discharge curves of the PPE$\mathrm{Fe}_{3} \mathrm{O}_{4}$ SC were studied to further evaluate the chargedischarge storage capacity of the PPE- $\mathrm{Fe}_{3} \mathrm{O}_{4} \mathrm{SC}$, as shown in Figure $3 \mathrm{~d}$. Consistent with the range of voltage in the $\mathrm{CV}$ curves in Figure $3 \mathrm{~b}$, the voltage of charge-discharge curves ranged from 0 to $0.6 \mathrm{~V}$, with current densities ranging from 0.22 to $0.75 \mathrm{~mA} \cdot \mathrm{cm}^{-2}$. The nearly symmetric curves indicated the good capacitive characteristics of the $\mathrm{PPE}-\mathrm{Fe}_{3} \mathrm{O}_{4} \mathrm{SC}$.

Figure $3 \mathrm{e}$ shows the Nyquist plot of the $\mathrm{PPE}-\mathrm{Fe}_{3} \mathrm{O}_{4} \mathrm{SC}$. Electrochemical impedance spectroscopy (EIS) was used to characterize the performance of the $\mathrm{PPE}-\mathrm{Fe}_{3} \mathrm{O}_{4} \mathrm{SC}$ at a frequency from $0.01 \mathrm{~Hz}$ to $100 \mathrm{kHz}$. The equivalent series resistance or internal resistance (ESR) of the PPE- $\mathrm{Fe}_{3} \mathrm{O}_{4} \mathrm{SC}$ calculated from the high-frequency intercept of the semicircle on the real axis was about $6.42 \Omega$, which was contributed by the resistance of electrolyte, the current collector, and also the contact resistance at the interface of the $\mathrm{PPE}-\mathrm{Fe}_{3} \mathrm{O}_{4}$ fiber. According to pre-experiment results, mixing oxide with EGaIn showed smaller ESR than that of attaching oxide on the surface of the PPE fibers. In addition, the PPE- $\mathrm{Fe}_{3} \mathrm{O}_{4}$ fiber not only were active materials but also replaced the metal plate as current collectors.

In addition, we measured the capacitance of the PPE- $\mathrm{Fe}_{3} \mathrm{O}_{4}$ $\mathrm{SC}$ over multiple charging and discharging cycles, as shown in Figure $3 \mathrm{f}$, because stable performance of a supercapacitor is required in actual applications. The $\mathrm{CV}$ response was measured to gain the change over 1000 cycles between -0.3 to $0.3 \mathrm{~V}$ at a scan rate of $200 \mathrm{mV} \cdot \mathrm{s}^{-1}$. The areal specific capacitance reduced to $92.51 \%$, and the CV curves remained symmetric after 1000 cycles, which indicated that the PPE$\mathrm{Fe}_{3} \mathrm{O}_{4} \mathrm{SC}$ had a long cycling life.

Figure 4 displays the electrochemical stability of the PPE$\mathrm{Fe}_{3} \mathrm{O}_{4}$ SC. In a practical application, supercapacitors need to adapt to various working conditions; therefore, it is necessary to measure the electrochemical stability of supercapacitors. With an increasing strain of $120 \%$, it can be seen that $97.08 \%$ of the specific capacitance was maintained, as shown in Figure 4 a. In fact, there is no significant reduction with the increase in strain up to $120 \%$. The optical photographs and CV curves are shown in Figure $4 \mathrm{~b}$ and Figure S5c when the PPE- $\mathrm{Fe}_{3} \mathrm{O}_{4} \mathrm{SC}$ was stretched to $200 \%$ and $150 \%$, respectively. In addition, the $\mathrm{CV}$ curves of the PPE- $\mathrm{Fe}_{3} \mathrm{O}_{4} \mathrm{SC}$ that was stretched under $50 \%$ strain and released for 100 cycles were measured. In Figure S5d we found that the CV curves are almost unchanged. As shown in Figure 4c, the specific capacitance decreased gradually when the bending angle increased, which retained $74.53 \%$ at least at the bending angle from 0 to $180^{\circ}$. The above study indicated that the $\mathrm{PPE}-\mathrm{Fe}_{3} \mathrm{O}_{4} \mathrm{SC}$ had superior electrochemical stability.

To demonstrate the power supply ability of the PPE- $-\mathrm{Fe}_{3} \mathrm{O}_{4}$ $\mathrm{SC}$, a charge-discharge experiment was conducted. As Figure $4 \mathrm{~d}$ shows, supercapacitors connected in series were charged by
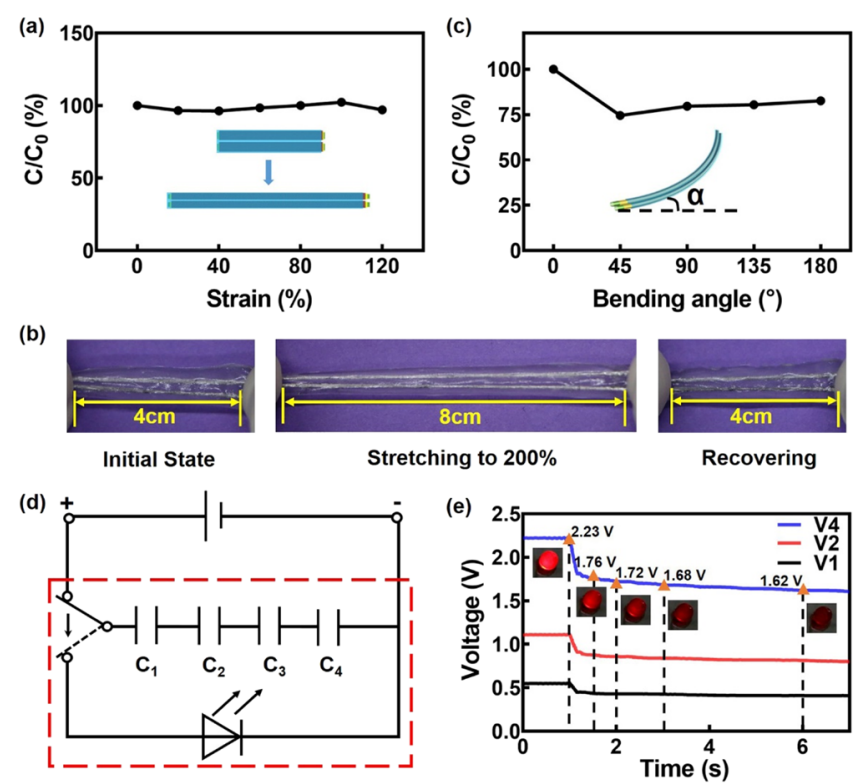

(f)

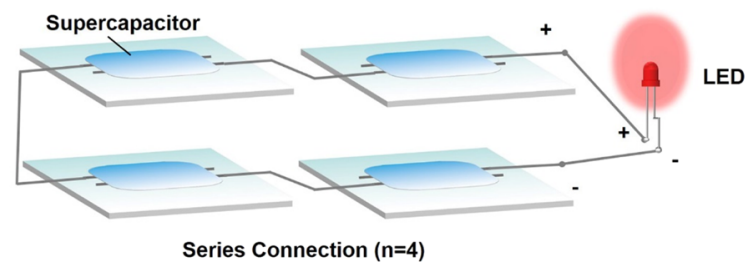

Figure 4. Performance of the PPE- $\mathrm{Fe}_{3} \mathrm{O}_{4}$ SC. (a) Evolution of specific capacitance over strains between 0 to $120 \%$, (b) photos of the PPE$\mathrm{Fe}_{3} \mathrm{O}_{4} \mathrm{SC}$ before, during, and after being stretched, and (c) evolution of specific capacitance over bending angles between 0 to $180^{\circ} . C_{0}$ and $C$ correspond to specific capacitances before and after stretching or bending, respectively. (d) Circuit diagram: charge the $\mathrm{PPE}-\mathrm{Fe}_{3} \mathrm{O}_{4} \mathrm{SCs}$ in series by a power source, and then cut off the power. (e) The voltage curve of the four SCs in series: V4 represents the voltage of four SCs, V2 represents the voltage of two SCs, and V1 represents the voltage of one SC. (f) The PPE- $\mathrm{Fe}_{3} \mathrm{O}_{4}$ SCs connected in series lighted the LED.

the electrochemical workstation to reach the operating voltage of the light-emitting diode (LED). Then, the power was cut off, and the LED was lighted by the supercapacitors. To get a better view of the LED shining, the whole process was carried out in a dark environment. Figure $4 \mathrm{e}$ shows the voltage curves of these supercapacitors in series. These curves correspond to voltages of one, two, and four capacitors. From Figure 4e, we can find that the voltage dividing between the four capacitors are basically equal. The total voltage of the supercapacitors was $2.23 \mathrm{~V}$ after being charged. When they were cut off from the electrochemical workstation and connected with the LED, the voltage decreased to $1.76 \mathrm{~V}$ within a short time, and the LED was lighted simultaneously. Afterward, a slow discharge process was observed. The LED was gradually dimming as time passed, as shown in Figure 4e. As expected, the $\mathrm{PPE}-\mathrm{Fe}_{3} \mathrm{O}_{4} \mathrm{SCs}$ exhibited a stable power supply effect.

\section{CONCLUSIONS}

In summary, this article puts forward a method to fabricate flexible supercapacitors based on PU@PMA@EGaIn fibers. As the circuit skeleton, PU fibers decided the construction of supercapacitors. EGaIn, as the electrode material, was outstanding for its excellent conductivity and fluidity. The 
introduction of $\mathrm{MnO}_{2}$ and $\mathrm{Fe}_{3} \mathrm{O}_{4}$ was to increase the surface area and store electricity by the effect of pseudo-capacitance, thus improving specific capacitance. Moreover, the gel electrolyte used in our experiment was $\mathrm{NaCl}-\mathrm{CaCl}_{2}$ PVA gel electrolyte. Its mechanical properties and ionic conductivity could be regulated through the ratio between $\mathrm{NaCl}$ and $\mathrm{CaCl}_{2}$, and the mixing ratio adopted was 2:3. The obtained PPE SCs were characterized and proven to have stable electrochemical and mechanical properties. At a scan rate of $5 \mathrm{mV} \cdot \mathrm{s}^{-1}$, the areal specific capacitances of the PPE- $\mathrm{Fe}_{3} \mathrm{O}_{4} \mathrm{SC}$ and the $\mathrm{PPE}-\mathrm{Fe}_{3} \mathrm{O}_{4}$ SC (vacuum) were 26.71 and $61.34 \mathrm{mF} \cdot \mathrm{cm}^{-2}$. The latter showed a larger specific capacitance through forming a coarser surface and airholes, but the capacitance was not steady. The former showed the best performance with stable specific capacitance and a strain of $120 \%$. Finally, the $\mathrm{PPE}-\mathrm{Fe}_{3} \mathrm{O}_{4}$ SCs were adopted to light the LED, demonstrating that those supercapacitors could work successfully. Advantages of the PPE SCs include a simple fabrication process, excellent flexibility, high areal specific capacitance, and a stable working performance. Future research may focus on the further improvement of the specific capacitance of supercapacitors based on liquid metal. Generally speaking, liquid metal is a promising candidate for flexible supercapacitors, which deserves more attention and effort in the near future.

\section{EXPERIMENTAL SECTION}

Preparation of EGaln. The EGaIn used in this experiment was composed of $75.5 \%$ gallium and $24.5 \%$ indium by weight. Gallium and indium were heated and stirred at $200{ }^{\circ} \mathrm{C}$ for $2 \mathrm{~h}$. After cooling down to room temperature, the alloy remained in the liquid state and EGaIn was formed.

Preparation of O-EGaln. EGaIn and metal oxide microparticles were placed into a beaker. They were stirred until EGaIn and the microparticles were intensively mixing. Finally, EGaIn in its liquid form turned into O-EGaIn in the paste form.

Preparation of $\mathrm{NaCl}-\mathrm{CaCl}_{2}$ PVA Gel Electrolyte. First, 5 $\mathrm{g}$ of PVA powder was added to $35 \mathrm{~mL}$ of deionized water at 95 ${ }^{\circ} \mathrm{C}$ and was stirred until PVA was sufficiently dissolved. Then, $2 \mathrm{~g}$ of $\mathrm{NaCl}$ and $3 \mathrm{~g}$ of $\mathrm{CaCl}_{2}$ were dissolved in deionized water separately and then added to the PVA solution. Finally, the mixture was stirred for $2 \mathrm{~h}$ to obtain $\mathrm{NaCl}-\mathrm{CaCl}_{2}$ PVA gel electrolyte.

Fabrication of PU@PMA@EGaln Fiber Electrodes. To improve the adhesion between EGaIn and PU fibers, PMA was smeared onto 20D PU fibers first. PU fibers were immersed in PMA solution for $20 \mathrm{~min}$ and then dried at $80^{\circ} \mathrm{C}$. Then, the PU@PMA fibers were soaked into an O-EGaIn bath pool, and EGaIn would uniformly adhere to the surface of the fibers to obtain PU@PMA@EGaIn fibers.

Supercapacitor Assembly. Two PU@PMA@EGaIn fibers were positioned parallel to each other and coated by $\mathrm{NaCl}-\mathrm{CaCl}_{2}$ PVA gel electrolyte. The whole system was then dried to vaporize excess water. At this point, the fabrication of soft PU@PMA@EGaIn supercapacitors was completed.

Characterization. SEM and EDS images were obtained using a scanning electron microscope and an energy dispersive spectrometer. To evaluate the electrochemical properties of PPE supercapacitors, we adopted a two-electrode system and an electrochemical workstation (CHI 660E, CH Instrument). Testing items included cyclic voltammetry, chronopotentiometry, and A.C. impedance. The voltage curve during the process of lighting the LED was recorded using a signal oscilloscope.

Calculation of the Electrochemical Performance. The specific capacitance was calculated from CV curves according to the following equation:

$$
C_{\text {sp }}=\frac{1}{2 \nu S V} \int_{V_{-}}^{V_{+}} i(V) \mathrm{d} V
$$

where $V_{+}$is the maximum voltage of the voltage window, $V_{-}$is the minimum voltage, $V\left(V_{+}-V_{-}\right)$is the width of the voltage window, $\nu$ is scan rate, and $S$ is the surface area.

\section{ASSOCIATED CONTENT}

\section{Supporting Information}

The Supporting Information is available free of charge at https://pubs.acs.org/doi/10.1021/acsomega.1c02834.

Preparation of O-EGaIn, SEM images of different fibers, and CV curves of PPE SC and PPE- $\mathrm{MnO}_{2} \mathrm{SC}$ (PDF)

\section{AUTHOR INFORMATION}

\section{Corresponding Author}

Jing Liu - Department of Biomedical Engineering, School of Medicine, Tsinghua University, Beijing 100084, China; Beijing Key Laboratory of Cryo-Biomedical Engineering, CAS Key Laboratory of Cryogenics, Technical Institute of Physics and Chemistry, Chinese Academy of Sciences, Beijing 100190, China; (1) orcid.org/0000-0002-0844-5296;

Email: jliubme@tsinghua.edu.cn

\section{Authors}

Minghui Duan - Department of Biomedical Engineering, School of Medicine, Tsinghua University, Beijing 100084, China

Yi Ren - Department of Biomedical Engineering, School of Medicine, Tsinghua University, Beijing 100084, China

Xuyang Sun - Beijing Key Laboratory of Cryo-Biomedical Engineering, CAS Key Laboratory of Cryogenics, Technical Institute of Physics and Chemistry, Chinese Academy of Sciences, Beijing 100190, China; School of Engineering Medicine and Interdisciplinary Institute for Cancer Diagnosis and Treatment, Beijing Advanced Innovation Center for Biomedical Engineering, Beihang University, Beijing 100191, China; (1) orcid.org/0000-0003-0867-1100

Xiyu Zhu - Department of Biomedical Engineering, School of Medicine, Tsinghua University, Beijing 100084, China

Xuelin Wang - School of Engineering Medicine and Interdisciplinary Institute for Cancer Diagnosis and Treatment, Beijing Advanced Innovation Center for Biomedical Engineering, Beihang University, Beijing 100191, China

Lei Sheng - Beijing Key Laboratory of Cryo-Biomedical Engineering, CAS Key Laboratory of Cryogenics, Technical Institute of Physics and Chemistry, Chinese Academy of Sciences, Beijing 100190, China

Complete contact information is available at:

https://pubs.acs.org/10.1021/acsomega.1c02834

\section{Author Contributions}

M.D. and Y.R. contributed equally to this work. M.D. contributed to conceptualization, methodology, investigation, data curation, and writing of the original draft of the study. Y.R. also contributed to the conceptualization, methodology, 
investigation, and writing of the original draft. X.S. contributed in designing the methodology, visualization, and funding acquisition. X.Z. participated in designing the methodology. X.W. acquired resources. L.S. contributed in funding acquisition. J.L. participated in review \& editing the paper, project administration, and funding acquisition.

\section{Funding}

This work was financially supported by the National Natural Science Foundation of China under Projects (nos. 51890893 and 81701850) and the China Postdoctoral Science Foundation (grant nos. 2018M641486 and 2020T130660).

\section{Notes}

The authors declare no competing financial interest.

\section{ACKNOWLEDGMENTS}

The authors thank Huimin Wang for his valuable discussions.

\section{REFERENCES}

(1) Sharma, P.; Bhatti, T. S. A review on electrochemical doublelayer capacitors. Energy Convers. Manage. 2010, 51, 2901-2912.

(2) Wang, C.; Wang, C.; Huang, Z.; Xu, S. Materials and structures toward soft electronics. Adv. Mater. 2018, 30, 1801368.

(3) Koo, J. H.; Song, J.; Kim, D. Solution-processed thin films of semiconducting carbon nanotubes and their application to soft electronics. Nanotechnology 2019, 30, 132001.

(4) Djenizian, T.; Tee, B.; Ramuz, M.; Fang, L. Advances in flexible and soft electronics. APL Mater. 2019, 7, No. 031201.

(5) Li, R.; Wang, L.; Yin, L. Materials and devices for biodegradable and soft biomedical electronics. Materials 2018, 11, 2108.

(6) Ngai, K. S.; Ramesh, S.; Ramesh, K.; Juan, J. C. A review of polymer electrolytes: fundamental, approaches and applications. Ionics 2016, 22, 1259-1279.

(7) Saeideh, A.; Saeedeh, M.; Seyed, H. A.; Farhad, S. Review of PVA-based gel polymer electrolytes in flexible solid-state supercapacitors: Opportunities and challenges. J. Energy Storage 2020, 27, 101072.

(8) Fard, H. N.; Pour, G. B.; Sarvi, M. N.; Esmaili, P. PVA-based supercapacitors. Ionics 2019, 25, 2951-2963.

(9) Sun, Z.; Yuan, A. Electrochemical performance of nickel hydroxide/activated carbon supercapacitors using a modified polyvinyl alcohol based alkaline polymer electrolyte. Chin. J. Chem. Eng. 2009, 17, 150-155.

(10) Imani, S.; Bandodkar, A. J.; Mohan, A. M. V.; Kumar, R.; Mercier, P. P. A wearable chemical-electrophysiological hybrid biosensing system for real-time health and fitness monitoring. Nat. Commun. 2016, 7, 11650.

(11) Becker, H. Low Voltage Electrolytic Capacitor. U.S. Patent 2,800,616A, 23(1957).

(12) Yassine, M.; Fabris, D. Performance of Commercially Available Supercapacitors. Energies 2017, 10, 1340.

(13) Lv, H.; Pan, Q.; Song, Y.; Liu, X.; Liu, T. A review on nano-/ microstructured materials constructed by electrochemical technologies for supercapacitors. Nano-Micro Lett. 2020, 12, 118.

(14) Brownson, D. A. C.; Kampouris, D. K.; Banks, C. E. An overview of graphene in energy production and storage applications. J. Power Sources 2011, 196, 4873-4885.

(15) A. B., Cultura, Z. M., Salameh. Performance evaluation of a supercapacitor module for energy storage applications. 2008 IEEE Power and Energy Society General Meeting - Conversion and Delivery of Electrical Energy in the 21st Century; IEEE: Pittsburgh, PA, USA(2008), pp. 1-7.

(16) Muralee Gopi, C. V. V.; Vinodh, R.; Sambasivam, S.; Obaidat, I. M.; Kim, H. J. Recent progress of advanced energy storage materials for flexible and wearable supercapacitor: from design and development to applications. J. Energy Storage 2020, 27, 101035.

(17) Choi, C.; Kim, S. H.; Sim, H. J.; Lee, J. A.; Choi, A. Y.; Kim, Y. T.; Lepró, X.; Spinks, G. M.; Baughman, R. H.; Kim, S. J. Stretchable, weavable coiled carbon nanotube/ $\mathrm{MnO}_{2}$ / polymer fiber solid-state supercapacitors. Sci. Rep. 2015, 5, 9387.

(18) Wang, H.; Wang, C.; Jian, M.; Wang, Q.; Xia, K.; Yin, Z.; Zhang, M.; Liang, X.; Zhang, Y. Superelastic wire-shaped supercapacitor sustaining $850 \%$ tensile strain based on carbon nanotube@ graphene fiber. Nano Res. 2017, 11, 2347-2356.

(19) Zhang, Y.; Bai, W.; Cheng, X.; Ren, J.; Weng, W.; Chen, P.; Fang, X.; Zhang, Z.; Peng, H. Flexible and stretchable lithium-ion batteries and supercapacitors based on electrically conducting carbon nanotube fiber springs. Angew. Chem., Int. Ed. 2015, 53, 1456414568.

(20) Chen, X.; Qiu, L.; Ren, J.; Guan, G.; Lin, H.; Zhang, Z.; Chen, P.; Wang, Y.; Peng, H. Novel electric double-layer capacitor with a coaxial fiber structure. Adv. Mater. 2013, 25, 6436-6441.

(21) Wu, Q.; Xu, Y.; Yao, Z.; Liu, A.; Shi, G. Supercapacitors based on flexible graphene/polyaniline nanofiber composite films. ACS Nano 2010, 4, 1963-1970.

(22) Aval, L. F.; Ghoranneviss, M.; Pour, G. B. High-performance supercapacitors based on the carbon nanotubes, graphene and graphite nanoparticles electrodes. Heliyon 2018, 4, No. e00862.

(23) Yi, L.; Liu, J. Liquid metal biomaterials: A newly emerging area to tackle modern biomedical challenges. Int. Mater. Rev. 2017, 62, 415-440.

(24) Wang, Q.; Yu, Y.; Liu, J. Preparations, characteristics and applications of the functional liquid metal materials. Adv. Eng. Mater. 2017, 20, 1700781 .

(25) Ordonez, R.; Hayashi, C.; Torres, C.; Hafner, N.; Adleman, J.; Acosta, N.; Melcher, J.; Kamin, N. M.; Garmire, D. Conformal LiquidMetal Electrodes for Flexible Graphene Device Interconnects. IEEE Trans. Electron Dev. 2016, 63, 4018-4023.

(26) Li, Q.; Lin, J.; Liu, T.; Zheng, H.; Liu, J. Printed flexible thinfilm transistors based on different types of modified liquid metal with good mobility. Sci. China Inf. Sci. 2019, 62, 202403.

(27) Kim, T.; Kim, D.; Lee, B.; Lee, J. Soft and Deformable Sensors Based on Liquid Metals. Sensors 2019, 19, 4250.

(28) Jin, C.; Zhang, J.; Li, X.; Yang, X.; Li, J.; Liu, J. Injectable 3-D fabrication of medical electronics at the target biological tissues. Sci. Rep. 2013, 3, 3442.

(29) Chen, G.; Wang, H.; Guo, R.; Duan, M.; Zhang, Y.; Liu, J. Superelastic EGaIn composite fiber sustaining 500\% tensile strain with superior electrical conductivity for wearable electronics. ACS Appl. Mater. Interfaces 2020, 12, 6112-6118.

(30) Park, H.; Kim, J. W.; Hong, S. Y.; Lee, G.; Ha, J. S. A dynamically stretchable supercapacitor for powering an integrated biosensor in all-in-one textile system. ACS Nano 2019, 13, 1046910480.

(31) Kim, M.; Lee, B.; Li, M.; Noda, S.; Kim, C.; Kim, J.; Song, W.; Lee, S. W.; Brand, O. All-soft supercapacitors based on liquid metal electrodes with integrated functionalized carbon nanotubes. ACS Nano 2020, 14, 5659-5667.

(32) Liu, D.; Su, L.; Liao, J.; Reeja-Jayan, B.; Majidi, C. Rechargeable soft-matter EGaIn- $\mathrm{MnO}_{2}$ battery for stretchable electronics. Adv. Energy Mater. 2019, 9, 1902798.

(33) Han, J.; Xu, C.; Zhang, J.; Xu, N.; Yao, X.; Cao, X.; Liang, Y.; Zheng, L.; Sun, J.; Zhai, J.; Sun, Q.; Wang, Z. Multifunctional coaxial energy fiber toward energy harvesting, storage, and utilization. ACS Nano 2021, 15, 1597-1607.

(34) Guo, R.; Tang, J.; Dong, S.; Lin, J.; Wang, H.; Liu, J.; Rao, W. Flexible Electronics: One-Step Liquid Metal Transfer Printing: Toward Fabrication of Flexible Electronics on Wide Range of Substrates. Adv. Mater. Technol. 2018, 3, 1870045.

(35) Peng, L.; Peng, X.; Liu, B.; Wu, C.; Xie, Y.; Yu, G. Ultrathin two-dimensional $\mathrm{MnO}_{2}$ /graphene hybrid nanostructures for highperformance, flexible planar supercapacitors. Nano Lett. 2013, 13, 2151-2157.

(36) Wang, H.-Y.; Xiao, F.-X.; Yu, L.; Liu, B.; Lou, X. W. Hierarchical $\alpha-\mathrm{MnO}_{2}$ nanowires@ $\mathrm{Ni}_{1-\mathrm{x}} \mathrm{Mn}_{\mathrm{x}} \mathrm{O}_{\mathrm{y}}$ nanoflakes core-shell nanostructures for supercapacitors. Small 2014, 10, 3181-3186. 